\title{
MyD88 in lung resident cells governs airway inflammatory and pulmonary function responses to organic dust treatment
}

\author{
Jill A. Poole ${ }^{1 *}$, Todd A. Wyatt ${ }^{1,2,3}$, Debra J. Romberger ${ }^{1,2}$, Elizabeth Staab ${ }^{1}$, Samantha Simet ${ }^{1}$, Stephen J. Reynolds ${ }^{4}$, \\ Joseph H. Sisson ${ }^{1}$ and Tammy Kielian ${ }^{5}$
}

\begin{abstract}
Inhalation of organic dusts within agriculture environments contributes to the development and/or severity of airway diseases, including asthma and chronic bronchitis. MyD88 KO (knockout) mice are nearly completely protected against the inflammatory and bronchoconstriction effects induced by acute organic dust extract (ODE) treatments. However, the contribution of MyD88 in lung epithelial cell responses remains unclear. In the present study, we first addressed whether ODE-induced changes in epithelial cell responses were MyD88-dependent by quantitating ciliary beat frequency and cell migration following wounding by electric cell-substrate impedance sensing. We demonstrate that the normative ciliary beat slowing response to ODE is delayed in MyD88 KO tracheal epithelial cells as compared to wild type (WT) control. Similarly, the normative ODE-induced slowing of cell migration in response to wound repair was aberrant in MyD88 KO cells. Next, we created MyD88 bone marrow chimera mice to investigate the relative contribution of MyD88-dependent signaling in lung resident (predominately epithelial cells) versus hematopoietic cells. Importantly, we demonstrate that ODE-induced airway hyperresponsiveness is MyD88-dependent in lung resident cells, whereas MyD88 action in hematopoietic cells is mainly responsible for ODE-induced TNF-a release. MyD88 signaling in lung resident and hematopoietic cells are necessary for ODE-induced IL-6 and neutrophil chemoattractant (CXCL1 and CXCL2) release and neutrophil influx. Collectively, these findings underscore an important role for MyD88 in lung resident cells for regulating ciliary motility, wound repair and inflammatory responses to ODE, and moreover, show that airway hyperresponsiveness appears uncoupled from airway inflammatory consequences to organic dust challenge in terms of MyD88 involvement.
\end{abstract}

\section{Introduction}

Inhalation of organic dusts can result in several inflammatory respiratory diseases, such as asthma, chronic bronchitis, obstructive lung disease, and hypersensitivity pneumonitis, particularly in agriculture-exposed workers [1]. The impact of agriculture exposures in respiratory diseases has recently become a central focus due to its association with increased risk of non-allergic mediatedairway hyper-responsiveness (AHR) and neutrophildominant inflammation, whereas providing apparent protection against the development of allergy and eosinophil mediated-asthma [2]. Moreover, currently available

\footnotetext{
* Correspondence: japoole@unmc.edu

${ }^{1}$ Pulmonary, Critical Care, Sleep \& Allergy Division, Department of Internal Medicine, University of Nebraska Medical Center, 985990 Nebraska Medical Center, Omaha, NE 68198-5990, USA

Full list of author information is available at the end of the article
}

therapeutics, including corticosteroids, lack efficacy in the treatment of agriculture-related respiratory diseases [3], underscoring the need to better understand the mechanisms governing the airway inflammatory and pulmonary function response to these environmental exposures.

Organic dusts from agriculture environments are now recognized to be comprised of an abundant and wide diversity of microbial motifs derived from both gram-positive and gram-negative bacteria [1]. Airway inflammatory consequences induced by organic dusts are partially reduced in mice deficient in Toll-like receptor 2 (TLR2) as well as TLR4, but organic dust-induced AHR is not affected by these pathways [4-6]. However, we previously reported a pivotal role for the TLR/IL-1R/IL-18R adaptor protein myeloid differentiation factor 88 (MyD88) that is used by all TLRs (except TLR3) in mediating the lung response to 
complex organic dusts [4]. Namely, acute organic dust extract (ODE)-induced AHR in conjunction with neutrophil influx and release of inflammatory cytokines/chemokines into the bronchoalveolar space, but not lung parenchyma, was nearly completely abrogated in MyD88 knock-out (KO) mice [4]. Of note, in comparison studies, we did not find a role for IL-1R, and only a limited role for IL-18R, signaling pathway in mediating acute ODE-induced airway inflammatory responses [4]. Based on these findings, we had speculated that MyD88-dependent signaling in lung resident cells, mainly epithelial cells, was mediating airway inflammatory and pulmonary function consequences to acute ODE challenge. However, the importance of MyD88 in epithelial cells and the relative contribution of MyD88 in the lung resident (i.e. epithelial) cell compartment as compared to leukocytes following organic dust exposures remained undefined.

In the present study, we first hypothesized that ODEmediated decreases in airway epithelial cilia beating and wound repair are mediated by MyD88-dependent signaling. To test this hypothesis, we utilized primary tracheal epithelial cells from MyD88 KO and wild type (WT) animals to compare ciliary motility and cell migration/wound repair responses following ODE treatment. Next, we hypothesized that acute ODE-induced AHR and airway inflammatory consequences were primarily mediated through MyD88-dependent signaling in airway epithelial cells. To test this hypothesis, we investigated respiratory mechanics through invasive pulmonary function testing as well as determined inflammatory cytokine/chemokine production and neutrophil influx following ODE treatment in MyD88 bone marrow chimera mice. The bone marrow chimera approach allowed us to differentiate between MyD88-dependent lung compartment effects, which were broadly defined as lung resident/structural cells versus hematopoietic cells. Our findings highlight novel MyD88-dependent epithelial cell functional responses of ciliary motility and wound repair to organic dust treatments. Furthermore, bone marrow chimeras revealed that AHR to ODE is dependent on MyD88 in lung resident cells, but that inflammatory consequences generally involve both resident and bone marrow derived leukocytes.

\section{Methods}

\section{Organic dust extract}

Aqueous organic dust extract (ODE) was prepared from settled dust collected from horizontal surfaces $(\sim 3$ feet above floor) of swine confinement feeding operations and extracts were batched prepared utilizing previously described methods [7]. Briefly, dust (1 gm) was placed into sterile Hank's Balanced Salt Solution (10 ml; Sigma, St. Louis, MO), incubated at room temperature for $1 \mathrm{~h}$, centrifuged for $20 \mathrm{~min}$ at $2200 \mathrm{rpm}$, and the final supernatant was filter sterilized $(0.22 \mu \mathrm{m})$, a process that also removes coarse particles. Stock ODE was diluted to a final concentration (vol/vol) of $5 \%$ for in vitro studies and $12.5 \%$ for in vivo studies in sterile phosphate buffered saline (PBS; $\mathrm{pH}$ : 7.4; diluent). These respective concentrations have been previously shown to elicit optimal experimental outcomes in airway epithelial cells and mice [5, 8-10] and are well tolerated [10]. ODE (100\%) contains roughly $4 \mathrm{mg} / \mathrm{ml}$ of total protein as measured by nanodrop spectrophotometry (NanoDrop Technologies, Wilmington, DE). Endotoxin concentrations are routinely measured throughout experimental studies, and $100 \%$ ODE endotoxin concentrations ranged from 160 to $400 \mathrm{EU} / \mathrm{ml}$ as assayed using the limulus amebocyte lysate assay according to manufacturer's instruction (Sigma). Muramic acid levels, a molecular component of bacterial cell wall peptidoglycans, were determined by mass spectrometry [11] and were $70 \mathrm{ng} / \mathrm{mg}$.

\section{Animals}

All animal procedures were approved by the Institutional Animal Care and Use Committee at the University of Nebraska Medical Center (protocol \# 10-054-07) and are in accordance with NIH guidelines for the use of rodents. MyD88 gene knockout (KO) mice on C57BL/6 background expressing the CD45.2 allele were provided by Dr. S. Akira (Osaka, Japan). Age- and sex-matched C57BL/6 mice (The Jackson Laboratory, Bar Harbor, ME) were used as wild-type (WT) controls. For the generation of MyD88 bone marrow chimeras, B6/SJL mice that are congenic for the CD45 allele (CD45.1) on a C57BL/6 background were used as WT animals and were purchased from The Jackson Laboratories. Mice were used between 12 and 16 weeks of age for all organic dust exposure in vivo airway studies.

\section{Exposure murine model}

Using our established intranasal (i.n.) inhalation exposure animal model [10], mice received treatment with $50 \mu \mathrm{l}$ of sterile saline (PBS) or $12.5 \%$ ODE under light isoflurane sedation. No mice exhibited respiratory distress throughout the treatment period.

\section{Ciliary motility assay}

Tracheas from unexposed WT and MyD88 KO mice were removed [12], and actively beating ciliated cells from tracheal rings were observed and their motion quantified using Sisson-Ammons video analysis (SAVA; Ammons Engineering, Mt. Morris, MI), a phase-contrast microscopy and computerized frequency spectrum analysis, as previously described [13]. After baseline ciliary beat frequency $(\mathrm{CBF})$ determination, tracheal rings were stimulated with procaterol $(100 \mathrm{nM})$ for $30 \mathrm{~min}$ and CBF was again recorded. Rings were also treated with $5 \%$ ODE for up to $24 \mathrm{~h}$ with CBF monitoring. During ciliary measurements, tracheal rings were maintained at a constant 
temperature $\left(24 \pm 0.5^{\circ} \mathrm{C}\right)$ by a thermostatically controlled heated stage. Whole-field analysis was performed using software that analyzes the entire captured image of all ciliated cells in a given field. The digital sampling rate was set at 85 frames per second for all experiments. The number of motile cilia was also recorded for each digital video image to rule out selection bias as previously described [14].

\section{Protein kinase activity}

Protein kinase activity was determined in murine tracheal epithelial cells following in vivo treatment with intranasal inhalation of saline or $12.5 \%$ ODE at $6 \mathrm{~h}$ post-treatment [10]. Epithelial cell lysates were immediately placed in $50 \mathrm{mM}$ Tris- $\mathrm{HCl}$ (pH 7.4) lysis buffer with protease inhibitors, and then assayed for protein kinase $\mathrm{C}$ (PKCE, PKC $\alpha$ ) and protein kinase A (PKA) activity as previously described $[8,10]$. Protein kinase activity was expressed in relation to the total amount of cellular protein assayed as picomoles of phosphate incorporated per minutes per milligram $(\mathrm{pmol} / \mathrm{min} / \mathrm{mg})$.

\section{Epithelial cell barrier function}

Tracheal epithelial cells were harvested from untreated WT and MyD88 KO mice as previously described [15], and were grown to confluence on electric cell substrate impedance sensing (ECIS) 96-well plate arrays (8W1E; Applied Biophysics, Troy, NY) in serum-free medium (1:1: Ham's F-12 [Hyclone Laboratories, Logan, UT] and DMEM (Gibco, Thermo Scientific, Grand Island, NY). The ECIS system measures transepithelial resistance changes in real-time. Epithelial cell migration following wounding was conducted as previously described [15]. Briefly, an elevated field pulse of $1400 \mu \mathrm{A}$ at $32,000 \mathrm{~Hz}$ was applied for $20 \mathrm{~s}$ to epithelial cells that produced a uniform circular lesion of $250 \mu \mathrm{m}$ in size. Cell migration was tracked over a period of $72 \mathrm{~h}$. Epithelial cell impedance was measured at $4000 \mathrm{~Hz}$, normalized to its value at the initiation of data acquisition, and plotted as a function of time.

\section{Bone marrow chimera generation}

To generate MyD88 bone marrow chimeras, CD45 congenic B6/SJL mice were used that harbor a CD45.1 allele originating from the SJL strain, whereas the remainder of the genome is derived from C57BL/6 mice. These animals represent the WT strain since they express functional MyD88. MyD88 KO mice are on a C57BL/6 background and express the CD45.2 allele, which allows for the discrimination between donor- and recipient-derived leukocytes based on staining with antibodies specific for CD45.1 and $\mathrm{CD} 45.2$. The following radiation chimeras were generated in these experiments (donor bone marrow $\rightarrow$ irradiated recipient): $\mathrm{WT} \rightarrow \mathrm{WT}, \mathrm{KO} \rightarrow \mathrm{KO}, \mathrm{WT} \rightarrow \mathrm{KO}$ and $\mathrm{KO} \rightarrow \mathrm{WT}$. The experimental chimeras were WT $\rightarrow$ $\mathrm{KO}$ and $\mathrm{KO} \rightarrow \mathrm{WT}$, whereas the other two groups (WT $\rightarrow$ $\mathrm{WT}$ and $\mathrm{KO} \rightarrow \mathrm{KO}$ ) represented controls to rule out any non-specific effects of irradiation on measured responses. Reconstitution of irradiated MyD88 KO animals with bone marrow from WT recipients ensured that leukocytes (i.e. macrophages, neutrophils, lymphocytes) derived from the bone marrow expressed MyD88, whereas lung structural cells (predominately epithelial cells) do not express MyD88 as a result of their radiation resistance [16-18]. The procedure for bone marrow chimera generation was based on our previously published studies [19]. Briefly, bone marrow donor mice were euthanized with sodium pentobarbital and marrow was isolated from the long bones by flushing with sterile $1 \times$ PBS. Recipient mice were placed on antibiotic-supplemented water $(1 \mathrm{~g} / \mathrm{l}$ neomycin and $125 \mathrm{mg} / \mathrm{l}$ polymyxin) for 3 days prior to bone marrow transfer and subjected to irradiation (1000 rad) using a RS-2000 Biological System (Rad Source) to destroy the bone marrow. Within 4 to $7 \mathrm{~h}$ following irradiation, recipient mice received a retro-orbital dose of $2 \times 10^{7}$ bone marrow cells supplemented with $1 \times 10^{7}$ cells from the spleen to serve as an immediate source of immune cells. Engraftment was allowed to take place over an 8 week period and chimeric animals were maintained on antibiotic-supplemented water for the first 2 weeks to provide protection during transient immunocompromise. At 8 weeks post-transplant, chimeric mice were bled retro-orbitally and cells were stained for flow cytometric analysis using CD45.1 and CD45.2 antibodies (BD Biosciences, Franklin, Lakes, NJ). Only animals that displayed chimerism of greater than $90 \%$ were used in organic dust airway exposure studies.

\section{Invasive pulmonary function measurements}

Total lung resistance $\left(R_{L}\right)$ and dynamic compliance (Cdyn) was invasively assessed via a tracheostomy tube $3 \mathrm{~h}$ following i.n. inhalation of saline or ODE treatment as previously described using a computerized small animal ventilator (Finepointe, Data Sciences International, St. Paul, MN) $[4,10]$. Dose responsiveness to aerosolized methacholine $(0-48 \mathrm{mg} / \mathrm{ml})$ was obtained and reported.

\section{Bronchoalveolar lavage fluid cell and cytokine/chemokine analysis}

In separate animal studies independent of the invasive pulmonary function measurement studies, bronchoalveolar lavage (BAL) was achieved using $3 \times 1 \mathrm{ml} \mathrm{PBS}$, and the total cell number recovered from pooled lavages was enumerated and differential cell counts were determined from cytospin-prepared slides (Cytopro Cytocentrifuge, Wescore Inc, Logan, UT) stained with DiffQuick (Siemens, Newark, DE). Cell counts were used to determine the differential ratio of leukocytes with 200 cells per slide 
per mouse. TNF- $\alpha$, IL- 6 , keratinocyte chemoattractant (KC; CXCL1), and macrophage inflammatory protein-2 (MIP-2; CXCL2) were quantitated in the cell-free supernatant of the first lavage by ELISA kits (R\&D Systems) with sensitivities of $10.9,7.8,15.6$, and $7.8 \mathrm{pg} / \mathrm{ml}$, respectively.

\section{Histopathology}

After whole lung lavage, lungs were harvested, inflated with $1 \mathrm{ml}$ of $10 \%$ formalin at a pressure of $20 \mathrm{~cm} \mathrm{H}_{2} \mathrm{O}$ for 1 day while submerged in $10 \%$ formalin for optimal preservation of lung parenchymal architecture as previously described [7]. Fixed lung tissues were processed using standard methods, embedded in paraffin, and thin sections $(4-5 \mu \mathrm{M})$ were stained with hematoxylin and eosin.

\section{Statistical methods}

Data are presented as mean and standard error of mean (SEM). Statistical significance was assessed by one-way analysis of variance (ANOVA) and a two-tailed Mann-Whitney test, where appropriate. For methacholine dose-response curves, two-way ANOVA was applied because two independent variables are involved (i.e. treatment group and methacholine dose), followed by Bonferroni post hoc tests when group differences were significant $(P<0.05)$.
GraphPad (Version 5.02, La Jolla, CA) software was used. Significance was accepted at $p$-values $<0.05$.

\section{Results \\ MyD88 affects the normative ciliary motility slowing response to organic dust}

The baseline ciliary beat frequency $(\mathrm{CBF})$ in primary tracheal epithelial rings from WT and MyD88 $\mathrm{KO}$ mice was $16.23 \pm 0.58 \mathrm{~Hz}$ and $14.2 \pm 1.174$, respectively ( $n=4$ independent experiments performed in triplicate; Fig. 1a-b). We have previously demonstrated that ODE slows CBF [14], and these findings were confirmed in WT mice (Fig. 1a). However, the normative ODE-induced CBF slowing response was absent in MyD88 $\mathrm{KO}$ epithelial cells at 1 and $6 \mathrm{~h}$ post-exposure $(N=6$ independent experiments performed in triplicate; Fig. 1a). In comparison, a 30 min treatment with the $\beta 2$ agonist, procaterol (10 nM), increased CBF in both WT and MyD88 KO cells (Fig. 1b), which demonstrates a normative intact cyclic nucleotide-dependent cilia beat response to a nonTLR agonist in MyD88 KO epithelial cells. There was no difference in the number of motile cilia points (an indicator of total number of beating cilia) among groups (data not shown). These studies suggest that MyD88 signaling is important for the effects of ODE on ciliary motility function.
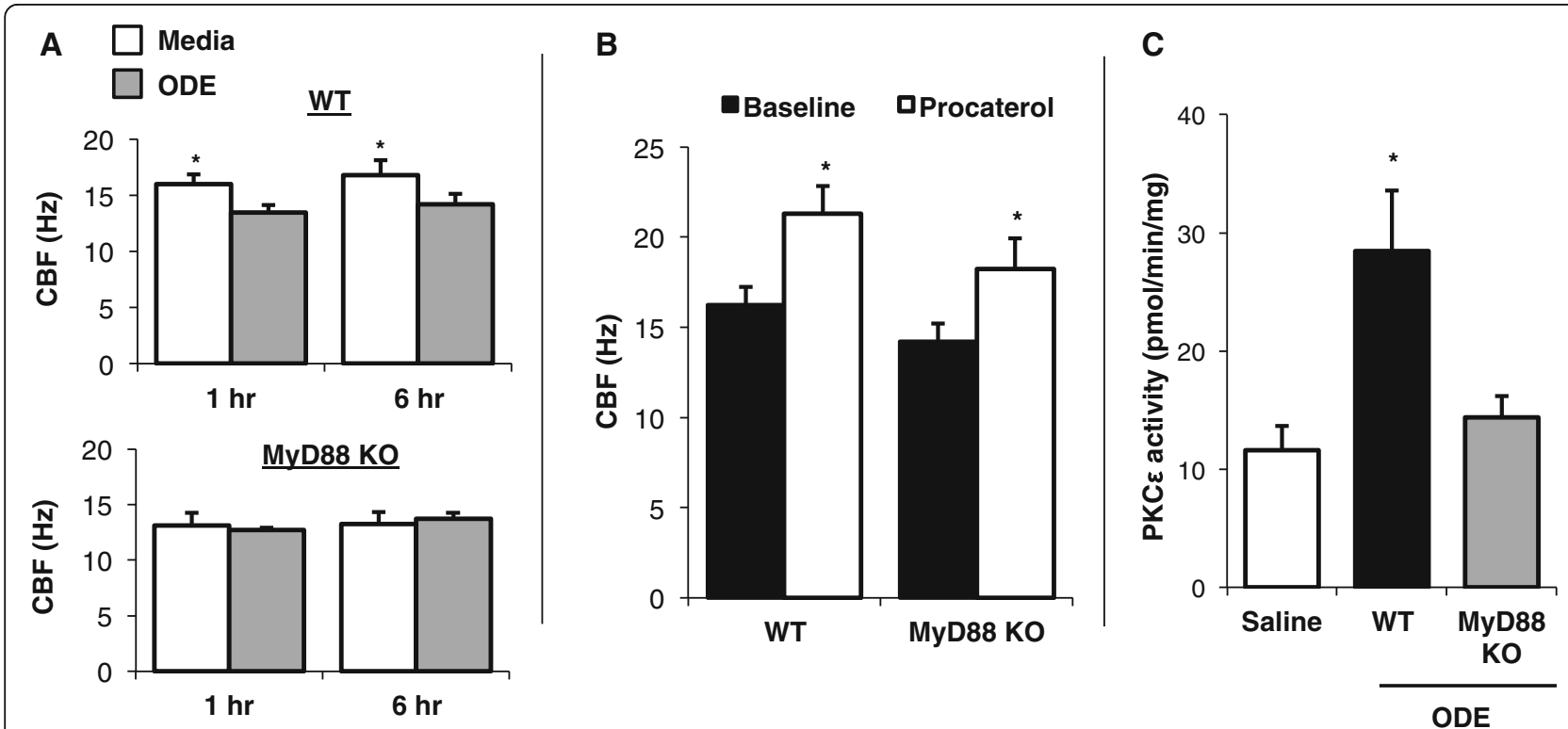

Fig. 1 The normative ciliary motility slowing response to ODE is absent in MyD88 KO epithelial cells. WT and MyD88 KO tracheal epithelial cells were investigated for ciliary beat frequency (CBF) by video recording and computer-generated calculations. a The normative CBF slowing response to $5 \%$ ODE is lost at 1 and $6 \mathrm{~h}$ in MyD88 KO cells. $\mathbf{b}$ The $\beta_{2}$ agonist, procaterol, increases CBF in WT and MyD88 KO epithelial cells, demonstrating intact cilia beat response in MyD88 KO cells to non-TLR agonist. Next, at $6 \mathrm{~h}$ post-intranasal inhalation of saline or ODE in WT and MyD88 KO mice, mice were euthanized and tracheal epithelial cells were harvested to determine protein kinase C (PKC) epsilon activity. c ODE-induced PKC $\varepsilon$ activity was absent in MyD88 KO epithelial cells. Bar graphs represent mean with SE bars shown of 4-6 independent experiments, each ran in triplicate. Statistical significance denoted by asterisk $\left({ }^{*} p<0.05\right)$ as compared to media control 
Next, we previously reported that ODE-induced slowing of $\mathrm{CBF}$ was dependent on ODE-induced $\mathrm{PKC} \varepsilon$ activity [14], and we also had demonstrated that ODE-induced $\mathrm{PKC} \varepsilon$ activity was absent in ODE-stimulated MyD88 KO lung slices [4]. In proof-of concept studies, WT and MyD88 KO animals were intranasal treated with saline or $12.5 \%$ ODE and at $6 \mathrm{~h}$ post-treatment, tracheal epithelial cells were harvested and protein kinase activity was determined. We confirm in primary tracheal epithelial cells that MyD88 signaling is important to ODE-induced PKCe activity because $\mathrm{PKC} \varepsilon$ activation was absent in MyD88 KO, but not WT, tracheal epithelial cells following in vivo ODE treatment (Fig. 1c). As a control, ODE inhalation treatment did not significantly alter PKC $\alpha$ or PKA activity at $6 \mathrm{~h}$ post-treatment in WT or MyD88 KO animals (data not shown).

\section{MyD88 KO epithelial cells demonstrate an aberrant} wound repair response to $O D E$

Cell migration and wound repair responses were determined using primary tracheal epithelial cells from WT and MyD88 KO mice following pretreatment with or without $5 \%$ ODE for $24 \mathrm{~h}$, whereupon cells were wounded using an automatic electric cell-substrate impedance sensing (ECIS) system. Cell migration data was continuously collected for up to $72 \mathrm{~h}$ post-wounding. Consistent with previous studies showing that ODE treatment slows wound closure [20], we demonstrated that ODE dampens cell migration and wound closure in WT epithelial cells (Fig. 2). However, in MyD88 KO cells, cell migration and wound repair responses were not significantly affected by ODE treatment over time (Fig. 2). A representative trace of one of five experimental studies is shown in Fig. 2a. Halfmaximal closure time (hr) (Fig. 2b) represent the mean time to which wounds were $50 \%$ closed among all five experiments. These data suggest that the wound repair response to ODE is dependent upon MyD88 signaling.

Bone marrow chimeras demonstrate that MyD88 in lung resident cells is important for regulating ODE-induced AHR and diminished dynamic compliance

Our previous work demonstrated a critical role for MyD88dependent pathways to acute ODE exposure in vivo because ODE-induced airway inflammation was nearly completely abrogated in MyD88 $\mathrm{KO}$ as compared to WT animals [4]. However, the importance of MyD88

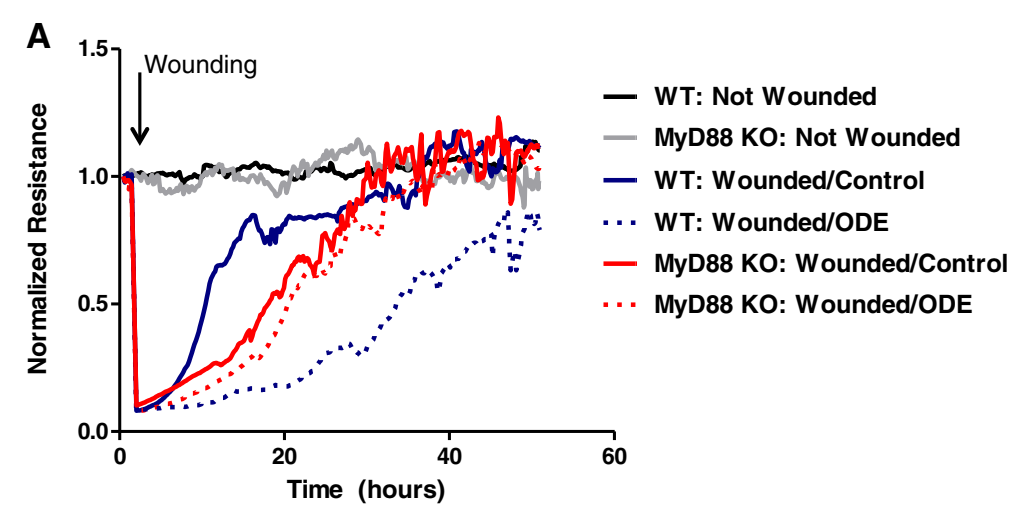

B

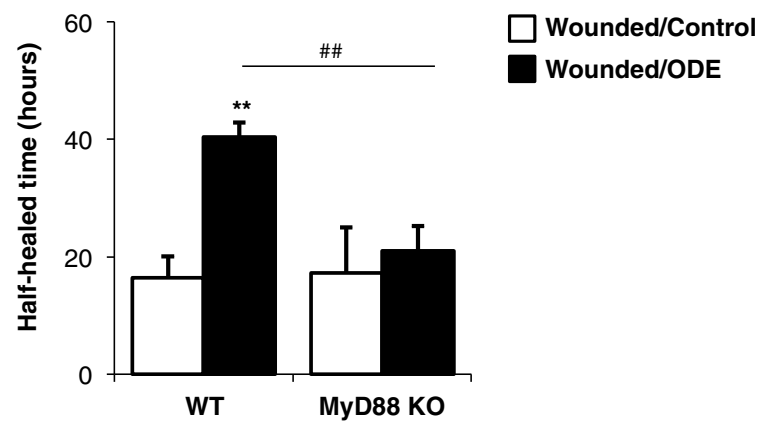

Fig. 2 MyD88 KO epithelial cells demonstrate an aberrant wound repair response to ODE. Cell migration (wound repair) response to ODE was assessed in WT and MyD88 KO tracheal epithelial cells by electric cell-substrate impedance sensing (ECIS) system. Cells were treated $\pm 5 \%$ ODE for $24 \mathrm{~h}$ prior to wounding. Cell migration data was continuously collected for up to $72 \mathrm{~h}$ post-wounding and displayed as normalized resistance (ohms) (subsequent values divided by initial values). a A representative tracing of data from one of 5 independent studies is shown. $\mathbf{b}$ Bar graph depicts mean $( \pm$ SEM) time to half-maximal closure in hours ( $N=5$ independent studies). Statistical significance denoted by asterisk (** $p<0.01)$ as compared to media control and hatchmarks (\#\#p<0.01) denoted by line 
in lung cell compartments is not known, and if evident, could lead to the future design of targeted cellular approaches. To understand the role of lung structural (predominately epithelial cell) versus hematopoieticderived immune cells (including alveolar macrophages) to acute ODE treatment, we generated MyD88 chimeric mice as described in the Methods section. Discrimination between donor and recipient cells was determined by CD45.1 and CD45.2 expression on circulating leukocytes. Namely, the MyD88 WT B6.SJL congenic mice express the CD45.1 allele and the MyD88 KO mice express the CD45.2 allele. In pilot studies, the dose of ionizing radiation administered to recipient mice to ablate the bone marrow without inducing toxicity (death or lung pathology) was determined as 1000 rads (data not shown). At 8 weeks following bone marrow transfer, CD45.1 and CD45.2 expression on peripheral blood leukocytes was determined by FACS. A representative histogram of bone marrow chimeric mice is shown in Fig. 3. The two experimental groups of animals included the presence of MyD88 in lung structural cells, but not in hematopoietic cells (i.e. $\mathrm{KO} \rightarrow \mathrm{WT}$ ) and vice versa (i.e. $\mathrm{WT} \rightarrow \mathrm{KO}$ ). The other two groups (WT $\rightarrow \mathrm{WT}$ and $\mathrm{KO} \rightarrow \mathrm{KO}$ ) represented controls to rule out any nonspecific effects of irradiation on measured responses. We could verify that the experimental chimeras and the WT $\rightarrow$ WT inflammatory profiles of irradiated saline and ODE treated control groups obtained in the present study were similar to those observed in non-irradiated
MyD88 WT and KO mice as recently reported [4]. Any mice that demonstrated incomplete chimerism (i.e. greater than $10 \%$ residual recipient phenotype) were excluded from the study. We show in Additional file 1: Figure S1 the lung histopathology of experimental chimeras treated with saline and ODE. Histopathology changes were slightly more pronounced in WT- $>\mathrm{KO}$ treated mice as compared to $\mathrm{KO} \rightarrow \mathrm{WT}$ animals.

Airway hyper-responsiveness (AHR) following large animal facility organic dust exposure is a central characteristic observed in humans $[10,21]$ and modeled in mice [10]. In general, the mechanisms governing AHR to organic dust exposures are not known, but we previously reported that ODE-induced AHR was abrogated in MyD88 KO mice [4]. In this study, we hypothesized that there would be equal contribution from lung structural cells and hematopoietic-derived immune cells in mediating MyD88-dependent AHR because neutrophil recruitment [22] and TNF- $\alpha$ [23] have been implicated in mediating non-allergic AHR. We utilized standard total lung resistance $\left(R_{L}\right)$ as the main AHR measurement because it reflects both narrowing of the conducting airways and parenchymal viscosity [24]. We found that ODE-induced AHR was MyD88-dependent only in lung structural cells (Fig. 4b). Namely, in the WT $\rightarrow \mathrm{KO}$ experimental group, where MyD88 was absent in lung structural cells but not hematopoietic-derived immune cells, there was no evidence of ODE-induced AHR as compared to saline control (Fig. $4 \mathrm{~b}$ ). In contrast, in the

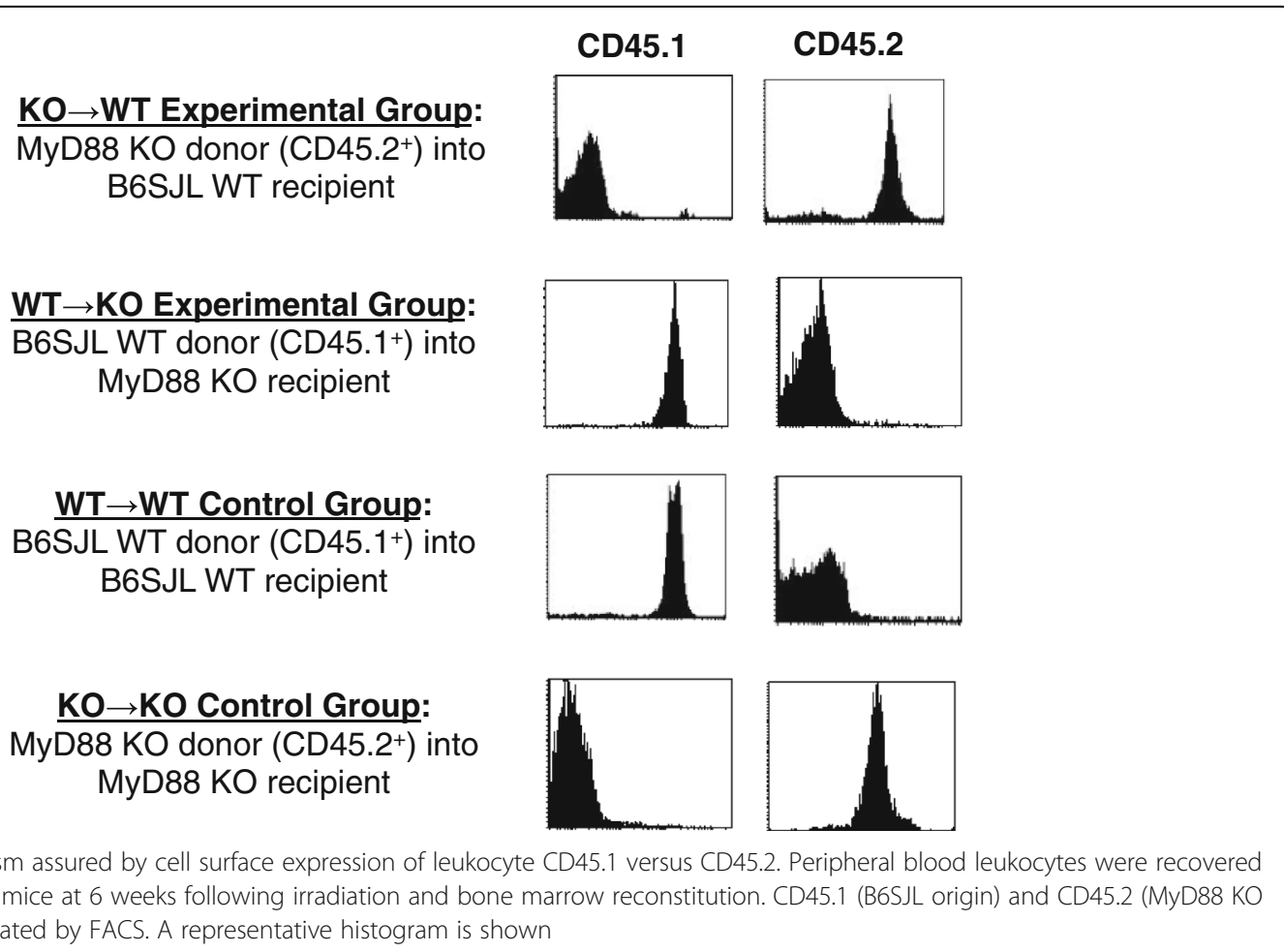



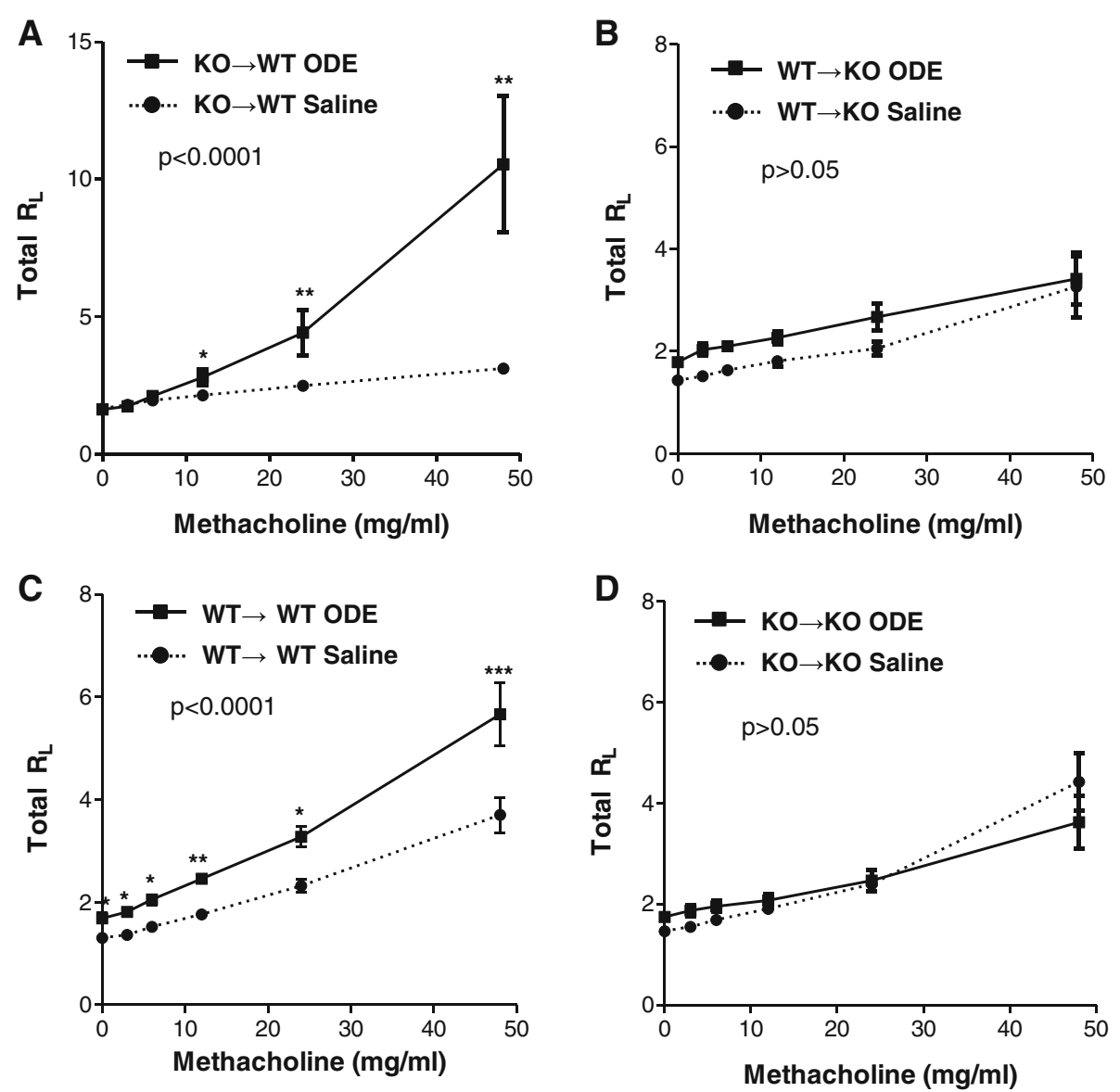

Fig. 4 MyD88 in lung structural cells is important for regulating ODE-induced airway hyper-responsiveness (AHR). MyD88 bone marrow chimeric mice (donor $\rightarrow$ recipient) were treated with ODE (solid line) or saline (dotted line), and at three hr following i.n. inhalation treatment mice were tracheostomized, mechanically ventilated, and AHR to aerosolized methacholine $(0,3,6,12,24,48 \mathrm{mg}$ ) was measure and expressed as mean ( \pm SEM) total lung resistance $\left(R_{L}\right)$. Chimeric groups depicted: a $\mathrm{KO} \rightarrow \mathrm{WT} ; \mathbf{b} \mathrm{WT} \rightarrow \mathrm{KO} ; \mathbf{c} W T \rightarrow W T ; \mathbf{d} \mathrm{KO} \rightarrow \mathrm{KO}$. Statistical difference between ODE and saline treatment groups was determined by two-way ANOVA (because there are 2 independent variables: treatment group and methacholine dose). If $p$-value less than 0.05 , differences between saline and ODE at each methacholine doses were determined by $t$-test and asterisks denote significance $\left({ }^{*} p<0.05,{ }^{* *} p<0.01,{ }^{* * *} p<0.001\right)$. $N=4-6$ mice/group

$\mathrm{KO} \rightarrow \mathrm{WT}$ group, where lung structural cells expressed MyD88 and the hematopoietic-derived immune cells lacked MyD88, ODE-induced AHR remained intact (Fig. 4a). Importantly, control groups demonstrated similar responses to ODE as in our prior report, showing lack of ODEinduced AHR in MyD88 KO mice [4], which established that irradiation did not significantly alter baseline lung responsiveness.

To broaden our understanding of how MyD88 affects lung function, we also investigated dynamic compliance (Cdyn), which reflects the elasticity of the lung parenchyma, but is also influenced by surface tension, smooth muscle contraction, and peripheral airway homogeneity [24]. First, in the WT $\rightarrow$ WT control group, ODE treatment decreased dynamic compliance as compared to saline (baseline measurement), and these differences remained with escalating concentrations of up to $24 \mathrm{mg}$ methacholine
(Fig. 5c). Similar to the WT $\rightarrow$ WT controls, the WT $\rightarrow$ $\mathrm{KO}$ experimental group where lung structural cells lack MyD88, displayed decreased dynamic compliance at baseline following ODE treatment (Fig. 5b), which was maintained with increasing doses of up to $24 \mathrm{mg}$ methacholine (Fig. 5b). In comparison, in the $\mathrm{KO} \rightarrow$ WT experimental group, where MyD88 was intact in lung resident cells, dynamic compliance differences between saline and ODE treatment were not observed until higher concentrations of methacholine were achieved (i.e. $24 \mathrm{mg}$ and $48 \mathrm{mg}$, Fig. 5a). There was no difference in dynamic compliance between saline and ODE treatments in the $\mathrm{KO} \rightarrow \mathrm{KO}$ control group (Fig. $5 \mathrm{~d}$ ). Collectively, these pulmonary function studies in chimera mice reveal a critical role for MyD88-dependent signaling in lung radiation-resistant, tissue-resident cells for mediating ODE-induced AHR and decreased dynamic compliance. 

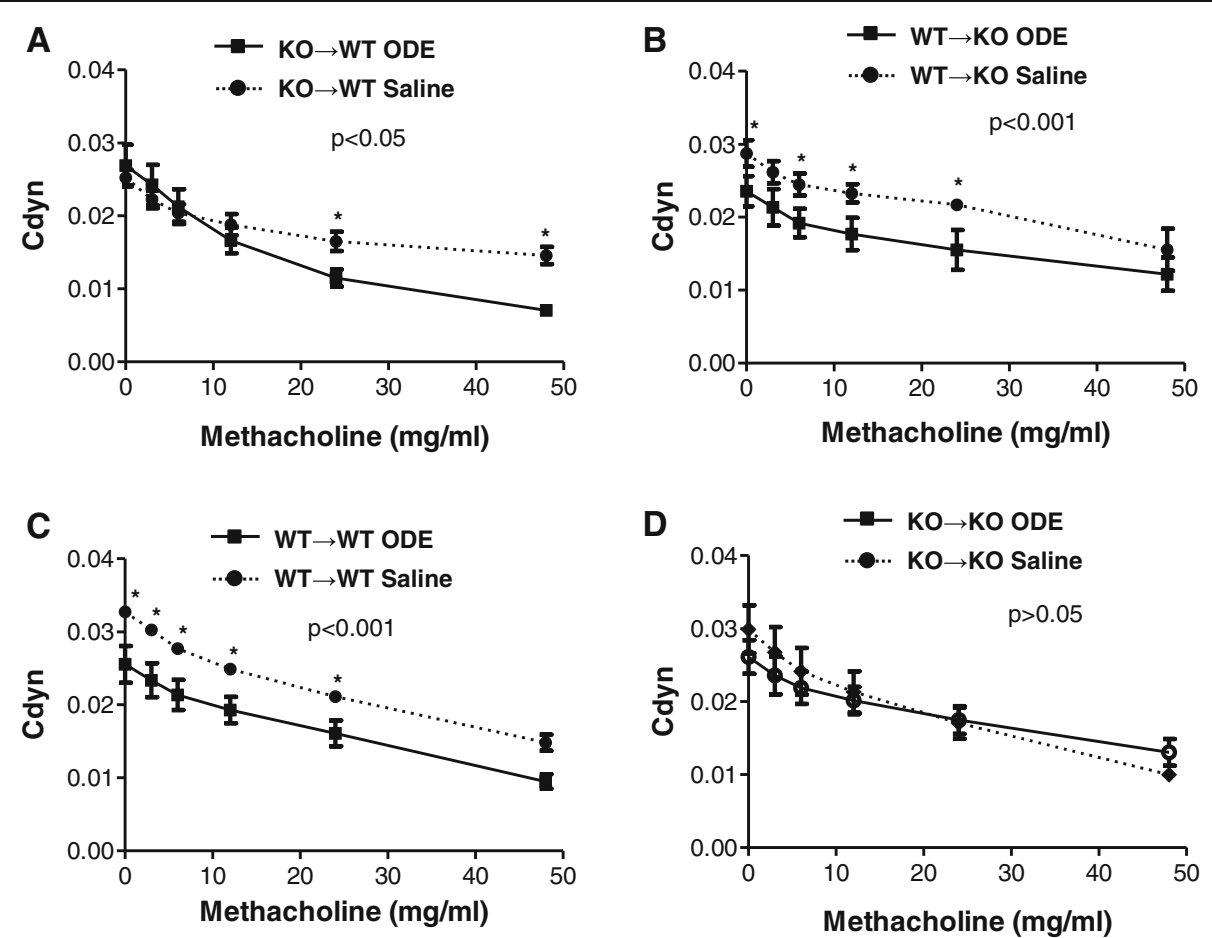

Fig. 5 ODE-induced decreased dynamic compliance is predominately dependent on MyD88 in lung structural cells. MyD88 bone marrow chimeric mice (donor $\rightarrow$ recipient) were treated with ODE (solid line) or saline (dotted line), and at three hr following i.n. inhalation treatment, mice were tracheostomized, mechanically ventilated, and dynamic compliance (Cdyn) following aerosolized methacholine (0, 3, 6, 12, 24, 48 mg) was measured and expressed as mean $( \pm \mathrm{SEM})$. Chimeric groups shown: a $\mathrm{KO} \rightarrow \mathrm{WT} ; \mathbf{b} \mathrm{WT} \rightarrow \mathrm{KO} ; \mathbf{c} W T \rightarrow W T ; \mathbf{d} \mathrm{KO} \rightarrow \mathrm{KO}$. Statistical difference between ODE and saline treatment groups was determined by two-way ANOVA (because there are 2 independent variables: treatment group and methacholine dose). If $p$-value less than 0.05 , differences between saline and ODE at each methacholine doses were determined by $t$-test and asterisks denote significance $\left.{ }^{*} p<0.05\right) . N=4-6$ mice/group

Important role of hematopoietic-derived immune cells for ODE-induced inflammatory cytokine/chemokine release Inflammatory mediators, particularly TNF- $\alpha$, have been suggested to mediate non-allergic AHR, and moreover, TNF- $\alpha$, IL-6, and CXCL8/IL-8 (murine homologs: CXCL1 and CXCL2) production have been demonstrated in organic dust-induced airway disease in humans and mice [25]. Consistent with our previous work demonstrating a critical role for MyD88 in the release of these mediators [4], ODE-induced mediator release was significantly diminished, but not completely abrogated, in the $\mathrm{KO} \rightarrow \mathrm{KO}$ control group as compared to the $\mathrm{WT} \rightarrow \mathrm{WT}$ control group (Fig. 6). Compared to saline treatment, IL-6 and CXCL2 were increased in the ODE-treated $\mathrm{KO} \rightarrow \mathrm{KO}$ control group (Fig. 6). The current studies were expanded to identify the relative importance of MyD88 signaling in the lung parenchymal vs. hematopoietic compartments, where cytokine/chemokine production in the BAL fluid from control and experimental chimeric animals was determined. We demonstrate that ODE-induced TNF- $\alpha$ production is primarily dependent upon MyD88 signaling in hematopoietic cells, since minimal TNF- $\alpha$ production was observed in the $\mathrm{KO} \rightarrow \mathrm{WT}$ experimental group (Fig. 6). This was corroborated by the finding that TNF- $\alpha$ production was nearly fully restored following WT bone marrow cell reconstitution of MyD88 KO mice (WT $\rightarrow$ KO; Fig. 6). We also demonstrate that CXCL1 and CXCL2 production is strongly, but not completely, dependent upon MyD88-signaling in hematopoietic-derived cells (Fig. 6). ODE-induced IL-6 production was dependent upon both MyD88 signaling in lung resident and hematopoietic cells (Fig. 6). Thus, ODE-induced TNF- $\alpha$, and to a lesser degree CXCL1 and CXCL2, release into the bronchoalveolar space is predominately dependent on MyD88 signaling from cells of bone marrow origin. In contrast, ODE-induced IL-6 production involves both MyD88-dependent lung radiation-resistant tissue resident and hematopoietic cells.

Both hematopoietic and resident lung cells are important for MyD88-dependent neutrophil influx into the airways following ODE treatment

Neutrophil influx is a hallmark of ODE-induced airway inflammatory responses; therefore, we next examined ODE-induced neutrophil influx in chimeric mice. As compared to the WT $\rightarrow \mathrm{WT}$ control group, the experimental groups $(\mathrm{KO} \rightarrow \mathrm{WT}$ and $\mathrm{WT} \rightarrow \mathrm{KO})$ and the $\mathrm{KO} \rightarrow$ $\mathrm{KO}$ control group demonstrated a significant reduction in 

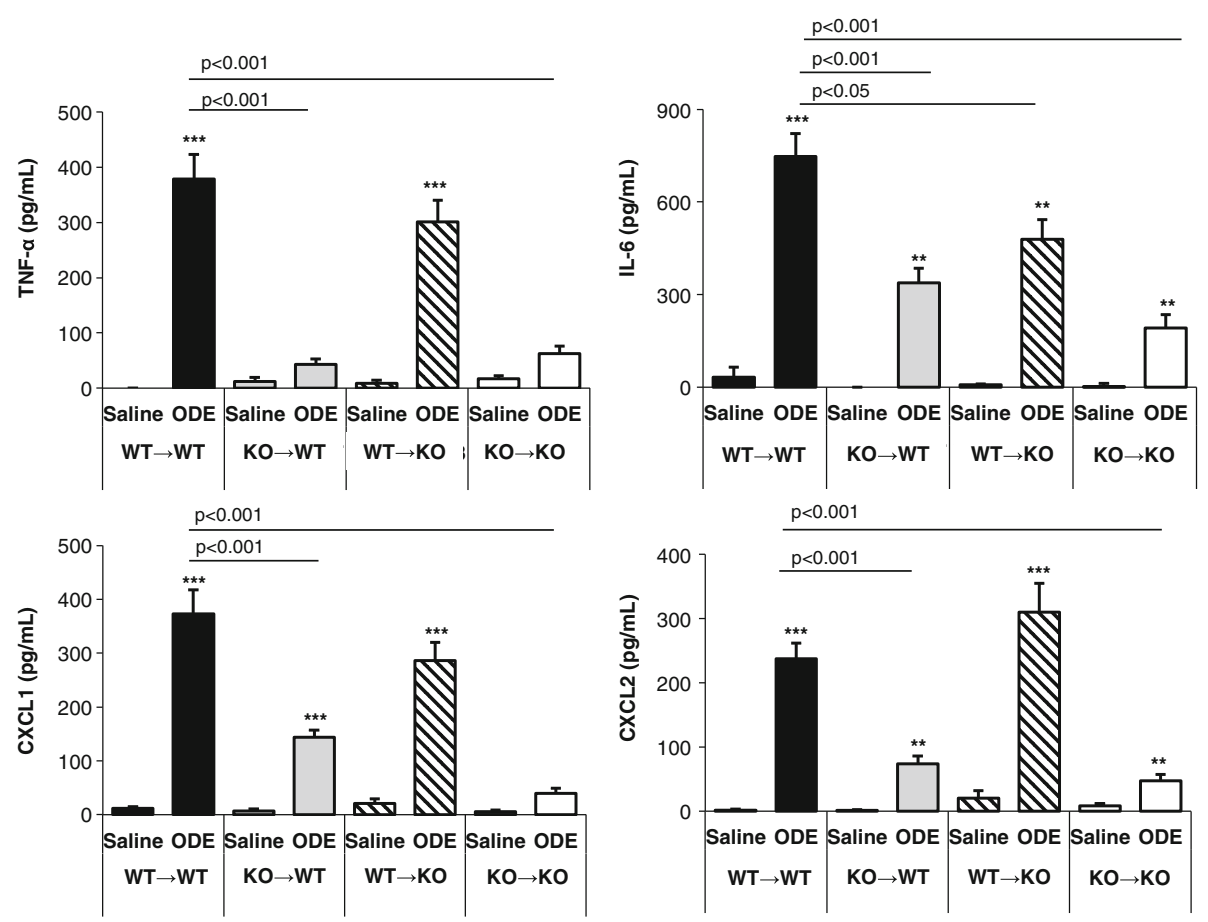

Fig. 6 Important role of hematopoietic cells mediating ODE-induced inflammatory cytokine/chemokine release. MyD88 bone marrow chimeric mice (donor $\rightarrow$ recipient) were treated once with ODE or saline and at five hr post treatment, TNF-a, IL-6 and the murine neutrophil IL-8 chemokine homologs (CXCL1 and CXCL2) were determined in BALF. The data represent mean with standard error bars shown ( $N=10-15$ mice per ODE; $N=4-8$ mice per saline treatment group). Statistical significance denoted by asterisks $\left(p<0.01,{ }^{* *} p<0.001\right)$ as compared to respective saline treatment group. Line denotes statistical significance of chimera mice to WT mice treated with ODE

ODE-induced neutrophil recruitment (Fig. 7). Of note, the $\mathrm{KO} \rightarrow \mathrm{KO}$ saline group showed a slight increase in neutrophil influx compared to the other saline treatments; however, this was not statistically significant (Fig. 7). Nonetheless, results from the experimental chimera groups indicate that efficient neutrophil recruitment following ODE exposure depends on MyD88 signals mediated through both lung resident and myeloid cells.

\section{Discussion}

Organic dusts from agriculture environments are complex mixtures containing a diverse array of microbial components and particulate matter that can rapidly activate airway inflammatory responses. Studies have shown partial reduction in organic dust-induced airway inflammation in TLR2-, TLR4-, and TLR9- deficient mice [4-6]. Moreover, IL-18R KO, but not IL-1R KO mice demonstrated slight reduction in airway inflammatory consequences to acute ODE treatment [4]. MyD88 is the common adaptor protein utilized by most TLRs (except TLR3) and IL-1R/IL-18R to transduce activation signals. We previously showed that AHR and inflammatory cell influx and mediator release into the bronchoalveolar space, but not lung parenchyma, following ODE challenge was nearly completely abrogated in MyD88-deficient animals [4]. We interpreted these findings to suggest a role for MyD88 in regulating epithelial cell

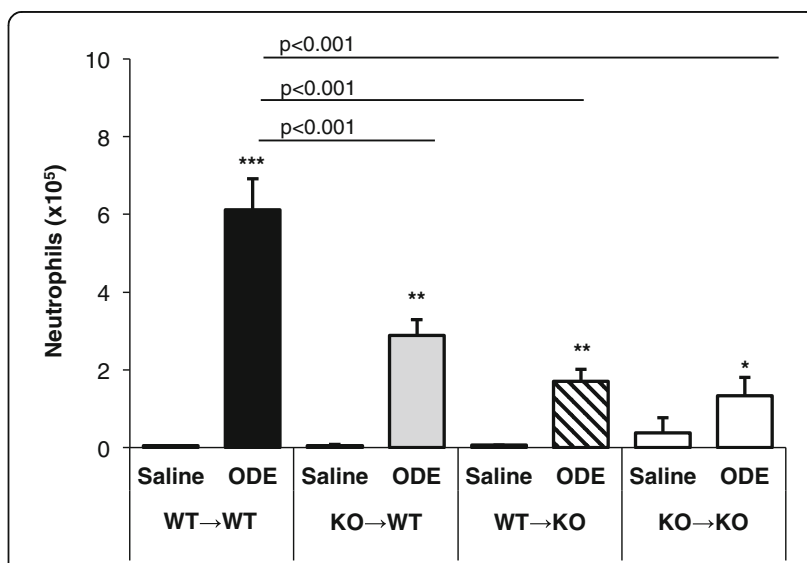

Fig. 7 Lung structural and hematopoietic cells are required for ODE-induced neutrophil influx. MyD88 bone marrow chimeric mice (donor $\rightarrow$ recipient) were treated once with ODE or saline. At five hr post treatment, neutrophil recruitment into the bronchoalveolar lavage fluid was determined. The data represent mean with standard error bars shown of $N=10-15$ mice per ODE and $N=4-8$ mice per saline treatment groups. Statistical significance denoted by asterisks $\left({ }^{*} p<0.05,{ }^{*} p<0.01,{ }^{* * *} p<0.001\right)$ as compared to respective saline treatment group. Line denotes statistical significance of chimera mice to WT mice treated with ODE 
inflammatory and repair consequences to complex organic dust exposures. However and in general, the role of MyD88 in epithelial cell functional was not well defined. Thus, our first objective of the current study was to define MyD88-dependent, epithelial cell responses following ODE exposures.

Mucociliary clearance and cellular migration/wound repair are important innate immune functions of epithelial cells that protect and repair the lung from deleterious effects of inhaled pollutants, allergens, and pathogens [26-28]. Exposure to cigarette smoke, bacterial pathogens, diesel exhaust particles, and organic dusts slow ciliary motility and decrease epithelial cell migration [14, 29-31]. Our studies demonstrate that MyD88 KO epithelial cells have normal baseline ciliary beat function and respond normally to the immunostimulatory non-TLR agent, procaterol (Fig. 1b). However, the ciliary motility slowing response to ODE treatment was blocked in MyD88 KO cells (Fig. 1a). The mechanisms to explain this response are likely due to the lack of a PKCE activation response in MyD88 KO cells. PKCe activation has been shown to mediate the ciliary slowing response to various environmental stimuli, including ODE [14]. Our previous data in MyD88 KO lung slices [4] and our current data in primary tracheal epithelial cells (Fig. 1c) support that ODE treatment slows $\mathrm{CBF}$ through $\mathrm{PKC} \varepsilon$ activation downstream of MyD88 signaling. Next, our studies demonstrate that MyD88 signaling is necessary to recognize and respond to wounding of epithelial cells to microbial componentenriched environmental dusts, suggesting a fundamental role for MyD88 in lung injury and repair responses. Others have also shown that MyD88 is required for normal resolution of epithelial injury due to sclerosing agent-induced tracheal insult [32].

We next addressed whether MyD88 signaling in the structural lung cell compartment was responsible for regulating pulmonary function in response to organic dust. Humans and mice that are exposed once to ODE or organic dust environments demonstrate significant increases in AHR and/or decreased cross-shift pulmonary function $[1,10,33-35]$. AHR to organic dust exposures are not explained by TLR2 or TLR4 action alone [5, 6], but can be completely ablated in MyD88 $\mathrm{KO}$ animals [4]. We expanded our earlier observations of a MyD88-dependent AHR response to ODE to clearly demonstrate here that ODE-induced AHR is fully dependent upon MyD88 signaling in the lung resident cell compartment as opposed to hematopoietic-derived cells (Fig. 4). We did not anticipate this strong delineation in lung compartment effects because others have suggested that non-allergic AHR can be mediated by neutrophils or TNF- $\alpha$ [22, 23]. Our studies do not support a role for either neutrophils or TNF- $\alpha$ in ODE-induced AHR. This is because MyD88-driven TNF- $\alpha$ production was dependent upon hematopoietic-derived immune cells and neutrophil recruitment was influenced by both compartments. However, our findings are consistent with LPS-induced bronchoconstriction, where LPSinduced non-invasive AHR measurements were found to be strictly dependent on MyD88 signaling by radiationresistant resident lung cells [36]. To our knowledge, the role of MyD88 signaling in airway smooth muscle and fibroblast function, independent of inflammatory cytokine production, has not been investigated, but might be important in the understanding of mechanisms and design of future therapies to target non-allergic mediated-AHR.

Asthma, hypersensitivity pneumonitis, and pulmonary fibrosis are associated with decreased lung dynamic compliance [37-40], and to varying degrees, these diseases have been reported in agriculture exposed humans $[1,41]$. However, general knowledge of how MyD88 effects lung compliance has been largely unexplored. Using bone marrow chimera mice, we demonstrate that MyD88 signaling by radiation-resistant resident lung cells is predominately responsible for mediating decreased dynamic compliance in response to ODE. Similar to ODE-induced AHR, changes in dynamic compliance cannot be completely explained by ODE-induced inflammatory consequences. Interestingly, others have recently reported that silicainduced fibrosis is uncoupled from silica-induced inflammation in MyD88 KO mice [42]. Collectively, these findings highlight that MyD88-dependent signaling in lung resident cells (i.e. epithelial, endothelial, smooth muscle, myofibroblast cells) may represent potentially targetable lung cell populations to impact the adverse respiratory mechanics following complex, organic dust exposures.

Acute exposure to organic dust environments rapidly results in TNF- $\alpha$, IL-6, and neutrophil chemoattractant (human CXCL8/IL-8 and murine CXCL1 and CXCL2) release. Our studies support that neutrophil influx and IL-6 production is dependent upon MyD88 signaling in both lung resident and hematopoietic-derived cells. TNF- $\alpha$ was almost fully dependent on MyD88-signaling in hematopoietic-derived immune cells. Release of the neutrophil chemoattractants (i.e. CXCL1 and CXCL2) was also predominately, but not entirely dependent on MyD88-signaling in hematopoietic immune cells. Although these studies were focused on delineating lung compartment-specific, MyD88-dependent functions, MyD88independent responses also exist because ODE-induced IL-6 and CXCL2 release and neutrophil influx, albeit significantly dampened, in the $\mathrm{KO} \rightarrow \mathrm{KO}$ control group (Figs. 6 and 7). Next, future studies could investigate the role of non-cytokine mediators, such as surfactants and phospholipids, in mediating ODE-induced airway hyperresponsiveness.

In summary, our data demonstrate a key role for MyD88 in important epithelial cell functions to complex organic dust exposures in vitro, including modifying ciliary motility 
and wound repair. Furthermore, we established a strong delineation in lung cell compartment effects, with respiratory mechanics dependent upon MyD88-mediated signaling in lung resident cells, likely epithelial cells, which is suggested by our in vitro studies where MyD88 KO epithelial cells were refractory to ODE-induced dysfunction. Both lung resident and hematopoietic-derived (i.e. myeloid) cells were responsible for efficient neutrophil recruitment, and inflammatory cytokine/chemokine production appears to be driven mostly by contributions from peripheral immune cells. This work provides evidence that lung inflammatory mediators induced by organic dust exposures are not responsible for mediating respiratory dysfunction consequences. These findings might have implications in the design of future therapeutic approaches for agriculture workers with lung disease. Namely, we would propose that future investigations into emerging therapeutic approaches that target epithelial damage and lung remodeling (i.e. stem cells, epithelial-mesenchymal cells) are warranted as potential new directions to reduce complex organic dust and/or environmental toxin-induced lung disease.

\section{Additional file}

Additional file 1: Figure S1. Lung parenchymal histopathology of experimental MyD88 bone marrow chimera (donor $\rightarrow$ recipient) mice treated once with saline or ODE. A representative murine lung section (hematoxylin and eosin stain, x10 magnification) is shown. Note that there is an absence of non-specific inflammation in saline-treated chimeric mice, and there is evidence of slight peribronchiolar and perivascular cellular cuffing following ODE treatment. Line scale is $100 \mu \mathrm{m}$. (PDF $699 \mathrm{~kb}$ )

\begin{abstract}
Abbreviations
AHR: Airway hyper-responsiveness; BALF: Bronchoalveolar lavage fluid; CBF: Ciliary beat frequency; COPD: Chronic obstructive pulmonary disease; CXCL1: Keratinocyte chemoattractant/KC; CXCL2: Macrophage inflammatory protein-2/MIP-2; ECIS: Electric cell substrate impedance sensing;

FACS: Fluorescence activated cell sorting; IL: Interleukin; KO: Knock out; MyD88: Myeloid differentiation factor 88; ODE: Swine confinement facility organic dust extract; PBS: Phosphate buffered saline; PKC: Protein kinase $C_{\text {; }}$ TLR: Toll-like receptor; TNF-a: Tumor necrosis factor-alpha; WT: Wild-type.
\end{abstract}

\section{Competing interests}

The authors declare that they have no competing interests.

\section{Authors' contributions}

JAP \& TK conceived of the study. JAP, TK, TAW and DJR contributed to the study design. ES contributed to all experimental studies. SS contributed to ECIS studies. SJR conducted organic dust component analysis. JHS contributed to ciliary beat studies. JAP drafted the initial draft of the manuscript. All authors contributed to its completion and all authors approve of the final manuscript.

\section{Acknowledgements}

The authors wish to thank Jane DeVasure, Christopher Bauer, Angela Gleason, and Jackie Pavlik for technical assistance and Lisa Chudomelka for manuscript preparation assistance. We also thank the UNMC Tissue Science Facility for assistance with lung tissue processing, sectioning, H\&E staining, and assistance with digital microscopy images prepared for the manuscript.

\section{Sources of funding}

Study supported by grants from the National Institute of Environmental Health Sciences (R01ES019325 to JAP), National Institute of Occupational
Safety Health (U54OH010162 to JAP and TAW and R01OH008539 to DJR), and National Institute of Alcohol Abuse and Alcoholism (R01AA008769 to JHS). This work was supported in part by the Central States Center for Agricultural Safety and Health (CS-CASH).

\section{Author details}

${ }^{1}$ Pulmonary, Critical Care, Sleep \& Allergy Division, Department of Internal Medicine, University of Nebraska Medical Center, 985990 Nebraska Medical Center, Omaha, NE 68198-5990, USA. ${ }^{2}$ Department of Environmental, Agricultural \& Occupational Health, University of Nebraska Medical Center, 985990 Nebraska Medical Center, Omaha, NE 68198-5990, USA. ${ }^{3}$ VA Nebraska-Western lowa Health Care System, Omaha, NE 68105, USA. ${ }^{4}$ High Plains Intermountain Center for Agricultural Health and Safety, Department of Environmental and Radiological Health Sciences, Colorado State University, Ft. Collins, CO, USA. ${ }^{5}$ Department of Pathology and Microbiology, University of Nebraska Medical Center, 985990 Nebraska Medical Center, Omaha, NE 68198-5990, USA.

Received: 11 May 2015 Accepted: 3 September 2015

Published online: 16 September 2015

\section{References}

1. May S, Romberger DJ, Poole JA. Respiratory health effects of large animal farming environments. J Toxicol Environ Health B Crit Rev. 2012;15(8):524-41.

2. Wells AD, Poole JA, Romberger DJ. Influence of farming exposure on the development of asthma and asthma-like symptoms. Int Immunopharmacol; 2014;23(1):356-63.

3. Strandberg K, Ek A, Palmberg L, Larsson K. Fluticasone and ibuprofen do not add to the effect of salmeterol on organic dust-induced airway inflammation and bronchial hyper-responsiveness. J Intern Med. 2008;264(1):83-94.

4. Bauer C, Kielian T, Wyatt TA, Romberger DJ, West WW, Gleason AM, et al. Myeloid differentiation factor 88-dependent signaling is critical for acute organic dust-induced airway inflammation in mice. Am J Respir Cell Mol Biol. 2013;48(6):781-9.

5. Poole JA, Wyatt TA, Kielian T, Oldenburg P, Gleason AM, Bauer A, et al. Toll-like receptor 2 regulates organic dust-induced airway inflammation. Am J Respir Cell Mol Biol. 2011:45(4):711-9.

6. Charavaryamath C, Juneau V, Suri SS, Janardhan KS, Townsend H, Singh B. Role of Toll-like receptor 4 in lung inflammation following exposure to swine barn air. Exp Lung Res. 2008;34(1):19-35.

7. Poole JA, Anderson L, Gleason AM, West WW, Romberger DJ, Wyatt TA. Pattern recognition scavenger receptor A/CD204 regulates airway inflammatory homeostasis following organic dust extract exposures. J Immunotoxicol. 2014.

8. Wyatt TA, Poole JA, Nordgren TM, DeVasure JM, Heires AJ, Bailey KL, et al. CAMP-dependent protein kinase activation decreases cytokine release in bronchial epithelial cells. Am J Physiol Lung Cell Mol Physiol. 2014;307(8):L643-51.

9. Wyatt TA, Slager RE, Heires AJ, Devasure JM, Vonessen SG, Poole JA, et al. Sequential activation of protein kinase $C$ isoforms by organic dust is mediated by tumor necrosis factor. Am J Respir Cell Mol Biol. 2010;42(6):706-15.

10. Poole JA, Wyatt TA, Oldenburg PJ, Elliott MK, West WW, Sisson JH, et al. Intranasal organic dust exposure-induced airway adaptation response marked by persistent lung inflammation and pathology in mice. Am J Physiol Lung Cell Mol Physiol. 2009;296(6):L1085-95.

11. Poole JA, Dooley GP, Saito R, Burrell AM, Bailey KL, Romberger DJ, et al. Muramic acid, endotoxin, 3-hydroxy fatty acids, and ergosterol content explain monocyte and epithelial cell inflammatory responses to agricultural dusts. J Toxicol Environ Health A. 2010;73(10):684-700.

12. Bailey KL, Bonasera SJ, Wilderdyke M, Hanisch BW, Pavlik JA, DeVasure J, et al. Aging causes a slowing in ciliary beat frequency, mediated by PKCepsilon. Am J Physiol Lung Cell Mol Physiol. 2014;306(6):L584-9.

13. Sisson JH, Stoner JA, Ammons BA, Wyatt TA. All-digital image capture and whole-field analysis of ciliary beat frequency. J Microsc. 2003;211 (Pt 2):103-11.

14. Wyatt TA, Sisson JH, Von Essen SG, Poole JA, Romberger DJ. Exposure to hog barn dust alters airway epithelial ciliary beating. Eur Respir J. 2008;31(6):1249-55.

15. Simet SM, Wyatt TA, DeVasure J, Yanov D, Allen-Gipson D, Sisson JH. Alcohol increases the permeability of airway epithelial tight junctions in Beas-2B and NHBE cells. Alcohol Clin Exp Res. 2012;36(3):432-42.

16. Couillin I, Vasseur V, Charron S, Gasse P, Tavernier M, Guillet J, et al. IL-1R1/ MyD88 signaling is critical for elastase-induced lung inflammation and emphysema. J Immunol. 2009;183(12):8195-202. 
17. Gasse P, Mary C, Guenon I, Noulin N, Charron S, Schnyder-Candrian S, et al. IL-1R1/MyD88 signaling and the inflammasome are essential in pulmonary inflammation and fibrosis in mice. J Clin Invest. 2007;117(12):3786-99.

18. Van Maele L, Fougeron D, Janot L, Didierlaurent A, Cayet D, Tabareau J, et al. Airway structural cells regulate TLR5-mediated mucosal adjuvant activity. Mucosal Immunol. 2014;7(3):489-500.

19. Garg S, Nichols JR, Esen N, Liu S, Phulwani NK, Syed MM, et al. MyD88 expression by CNS-resident cells is pivotal for eliciting protective immunity in brain abscesses. ASN Neuro. 2009; 1(2): 10.1042/AN20090004.

20. Slager RE, Allen-Gipson DS, Sammut A, Heires A, Devasure J, Von Essen SG, et al. Hog barn dust slows airway epithelial cell migration in vitro through a PKC\{alpha\}-dependent mechanism. Am J Physiol Lung Cell Mol Physiol. 2007.

21. Demanche A, Bonlokke J, Beaulieu MJ, Assayag E, Cormier Y. Swine confinement buildings: effects of airborne particles and settled dust on airway smooth muscles. Ann Agric Environ Med. 2009;16(2):233-8.

22. Savov JD, Gavett SH, Brass DM, Costa DL, Schwartz DA. Neutrophils play a critical role in development of LPS-induced airway disease. Am J Physiol Lung Cell Mol Physiol. 2002;283(5):L952-62.

23. Pennings HJ, Kramer K, Bast A, Buurman WA, Wouters EF. Tumour necrosis factor-alpha induces hyperreactivity in tracheal smooth muscle of the guinea-pig in vitro. Eur Respir J. 1998;12(1):45-9.

24. Glaab T, Taube C, Braun A, Mitzner W. Invasive and noninvasive methods for studying pulmonary function in mice. Respir Res. 2007;8:63.

25. Poole JA, Romberger DJ. Immunological and inflammatory responses to organic dust in agriculture. Curr Opin Allergy Clin Immunol. 2012;12(2):126-32.

26. Livraghi A, Randell $\mathrm{SH}$. Cystic fibrosis and other respiratory diseases of impaired mucus clearance. Toxicol Pathol. 2007:35(1):116-29.

27. Vignola AM, Mirabella F, Costanzo G, Di Giorgi R, Gjomarkaj M, Bellia V, et al. Airway remodeling in asthma. Chest. 2003;123(3 Suppl):417S-22.

28. Ganesan S, Sajjan US. Repair and Remodeling of airway epithelium after injury in Chronic Obstructive Pulmonary Disease. Curr Respir Care Rep. 2013; 2(3): 10.1007/s13665-013-0052-2.

29. Navarrette CR, Sisson JH, Nance E, Allen-Gipson D, Hanes J, Wyatt TA. Particulate matter in cigarette smoke increases ciliary axoneme beating through mechanical stimulation. J Aerosol Med Pulm Drug Deliv. 2012;25(3):159-68.

30. Bailey KL, LeVan TD, Yanov DA, Pavlik JA, DeVasure JM, Sisson JH, et al. Non-typeable Haemophilus influenzae decreases cilia beating via protein kinase Cepsilon. Respir Res. 2012; 13: 49-9921-13-49.

31. Wyatt TA, Sisson JH, Allen-Gipson DS, McCaskill ML, Boten JA, Devasure JM, et al. Co-exposure to cigarette smoke and alcohol decreases airway epithelial cell cilia beating in a protein kinase cepsilon-dependent manner. Am J Pathol. 2012;181(2):431-40.

32. Giangreco A, Lu L, Mazzatti DJ, Spencer-Dene B, Nye E, Teixeira VH, et al. Myd88 deficiency influences murine tracheal epithelial metaplasia and submucosal gland abundance. J Pathol. 2011;224(2):190-202.

33. Donham KJ, Cumro D, Reynolds SJ, Merchant JA. Dose-response relationships between occupational aerosol exposures and cross-shift declines of lung function in poultry workers: recommendations for exposure limits. J Occup Environ Med. 2000;42(3):260-9

34. Donham KJ, Reynolds SJ, Whitten P, Merchant JA, Burmeister L, Popendorf WJ. Respiratory dysfunction in swine production facility workers: dose-response relationships of environmental exposures and pulmonary function. Am J Ind Med. 1995;27(3):405-18.

35. Burch J, Svendsen E, Siegel P, Wagner S, Von Essen S, Keefe T, et al. Endotoxin exposure and inflammation markers among agricultural workers in Colorado and Nebraska. J Toxicol Environ Health. 2010;73(1):5-22.

36. Noulin N, Quesniaux VF, Schnyder-Candrian S, Schnyder B, Maillet I, Robert T, et al. Both hemopoietic and resident cells are required for MyD88-dependent pulmonary inflammatory response to inhaled endotoxin. J Immunol. 2005;175(10):6861-9.

37. Sciurba FC. Physiologic similarities and differences between COPD and asthma. Chest. 2004;126(2 Suppl):117S-24. discussion 159S-161S.

38. Mitchell HW, Turner DJ, Gray PR, McFawn PK. Compliance and stability of the bronchial wall in a model of allergen-induced lung inflammation. J Appl Physiol (1985). 1999;86(3):932-7.

39. Zielonka TM, Demkow U, Filewska M, Bialas B, Zycinska K, Radzikowska E, et al. Angiogenic activity of sera from extrinsic allergic alveolitis patients in relation to clinical, radiological, and functional pulmonary changes. Lung. 2010;188(5):375-80.
40. Shimabukuro DW, Sawa T, Gropper MA. Injury and repair in lung and airways. Crit Care Med. 2003;31(8 Suppl):S524-31.

41. Girard M, Cormier Y. Hypersensitivity pneumonitis. Curr Opin Allergy Clin Immunol. 2010;10(2):99-103.

42. Re SL, Yakoub Y, Devosse R, Uwambayinema F, Couillin I, Ryffel B, et al. Uncoupling between inflammatory and fibrotic responses to silica: evidence from MyD88 knockout mice. PLoS One. 2014;9(7), e99383.

\section{Submit your next manuscript to BioMed Central and take full advantage of:}

- Convenient online submission

- Thorough peer review

- No space constraints or color figure charges

- Immediate publication on acceptance

- Inclusion in PubMed, CAS, Scopus and Google Scholar

- Research which is freely available for redistribution

Submit your manuscript at www.biomedcentral.com/submit 